\title{
The Refugee Co-Location Model may be useful in addressing refugee barriers to care. What do refugees think?
}

\author{
Michael Au (iD) A,B,E, Robyn Preston (iD) B,C, Robin A. Ray (iD) B and Meg Davis (iD) D \\ A Western Sydney Local Health District, NSW, Australia. \\ ${ }^{\mathrm{B}}$ College of Medicine and Dentistry, James Cook University, Qld, Australia. \\ ${ }^{\mathrm{C}_{\text {School }}}$ of Health, Medical and Applied Sciences, Central Queensland University, QId, Australia. \\ Downsville Multicultural Support Group, Townsville, Qld, Australia. \\ ${ }^{\mathrm{E}}$ Corresponding author. Email: michael.au@my.jcu.edu.au
}

\begin{abstract}
Co-location of services for refugees may be beneficial in addressing barriers to care. This model of care involves support for a specialist refugee nurse service with general practice, as well as developing partnerships with settlement support agencies and Primary Health Networks. We consider published literature on refugee perceptions of colocation, different models of care, upcoming research and priorities in the area.
\end{abstract}

Keywords: models of care, service delivery, underserved populations, minority, regional, remote, disparity, multidisciplinary.

Received 27 July 2021, accepted 4 November 2021, published online 23 November 2021

We read with great interest Sackey et al.'s (2020) integrated model of healthcare to deliver specialist refugee services in primary healthcare settings, published in Volume 26 Issue 6 of the Australian Journal of Primary Health. The Co-location Model, as described by Sackey et al. (2020), involves support for a specialist refugee nurse service with general practice, as well as developing partnerships with settlement support agencies and Primary Health Networks (PHNs), to deliver care for refugees. This model of care has been in place for some years in South East Queensland, comparable to other models of care in Victoria, but sparse in regional settings, despite ongoing focus of the Australian Government to resettle refugees in these regions (Department of Social Services 2018). Co-location of services may be a model of care more suited to regional settings than centralised specialist refugee health centres that are readily accessible in metropolitan settings (Milosevic et al. 2012).

Co-location of services in primary health is important. It breaks down accessibility barriers, where access to specialist resources dedicated for refugee health, such as on-site interpreting, psychiatry, and counselling, and infectious disease specialists, can all be utilised in a multi-disciplinary setting to enhance communication and continuity of care. Services play important roles in addressing familiarity and promoting information sharing practices for refugees (Au et al. 2019), which can be enhanced with co-located services. In addition, access to onsite interpreting may be particularly useful for specialist and allied health services, as they currently do not have free access to the Translating and Interpreting Service, which is only freely available to general practice.

Refugee perceptions of co-location of services have been described in some papers. Cheng et al. (2015), Owens et al.
(2016) and Valibhoy et al. (2017) described refugee and settlement worker perceptions, favouring the co-location of different healthcare services to avoid the necessity of multiple trips, difficulty with transport and navigating the healthcare system. McBride et al. (2017) described the Monash Health Refugee Health and Wellbeing Service Model (MHRHW), comprising of primary health care, co-located specialist tertiary services, refugee health nurses, allied health and capacity-building secondary consultation staff. Users of the service regarded the ability to attend multiple services at the one site as an important benefit. We wonder how these models of co-location differ between states, how they adapt to specific contexts and how outcomes might differ.

We believe the success of the Co-location Model will rely strongly on the commitment of general practitioners and PHNs to refugee health. Presently, there is no nationally coordinated policy on refugee health. Addressing refugee health has largely been left to responsibilities of the states and community interventions. However, the role of government would be to promote, coordinate, and incentivise this commitment to refugee health. Refugee health nurses, community health workers, PHNs and settlement organisations can also play significant roles in building capacity of service organisations that are committed to refugee health (Timlin et al. 2020; Wei et al. 2021). A recently published pragmatic stepped-wedge cluster randomised trial, the OPTIMISE study, demonstrated modest increases in the proportion of 14633 refugee patients undergoing comprehensive health assessment from 31 general practices in metropolitan Sydney and Melbourne. Facilitators from local health services worked with private practice teams to improve the organisation and delivery of services to refugees. This model of capacity 
building through outreach facilitation in primary health settings is an interesting framework and may be applicable in regional or remote settings where permanent co-location may be more challenging to implement. However, more information is needed on whether longer-term or more intensified partnerships would have greater impacts (Russell et al. 2021). Service provider barriers to delivery of refugee healthcare have been well described in the literature and will need to be addressed, such as issues of renumeration, interpreting and training support (Johnson et al. 2008; Farley et al. 2014; Harding et al. 2019).

Indeed, any model of care will need to adapt to regional contexts with different refugee profiles. Adapting to the language and cultural needs of different refugee communities requires careful consideration. Fostering partnerships between organisations and private practices will require time. Local barriers will also need to be addressed.

We thank Sackey et al. (2020) for their valuable contribution to the literature and their continued work at Mater Refugee Health Service and Refugee Health Network Queensland.

\section{Data availability statement}

Data sharing is not applicable as no new data were generated or analysed during this study.

\section{Conflicts of interest}

Robyn Preston is an Associate Editor with the Australian Journal of Primary Health. The authors declare no other conflicts of interest.

\section{Declaration of funding}

This research did not receive any specific funding.

\section{References}

Au M, Anandakumar AD, Preston R, Ray RA, Davis M (2019) A model explaining refugee experiences of the Australian healthcare system: a systematic review of refugee perceptions. BMC International Health and Human Rights 19, 22. doi:10.1186/s12914-019-0206-6

Cheng IH, Vasi S, Wahidi S, Russell G (2015) Rites of passage: improving refugee access to general practice services. Australian Family Physician 44, 503-507.
Department of Social Services (2018) 'Humanitarian Settlement in Regional Australia.' Available at https://immi.homeaffairs.gov.au/settling-inaustralia/humanitarian-settlement-program/about-the-program/humanitariansettlement-regional-australia [Verified 17 July 2021]

Farley R, Askew D, Kay M (2014) Caring for refugees in general practice: perspectives from the coalface. Australian Journal of Primary Health 20, 85-91. doi:10.1071/PY12068

Harding C, Seal A, Duncan G, Gilmour A (2019) General practitioner and registrar involvement in refugee health: exploring needs and perceptions. Australian Health Review 43, 92-97. doi:10.1071/AH17093

Johnson DR, Ziersch AM, Burgess T (2008) I don't think general practice should be the front line: experiences of general practitioners working with refugees in South Australia. Australia and New Zealand Health Policy 5, 20. doi: 10.1186/1743-8462-5-20

McBride J, Block A, Russo A (2017) An integrated healthcare service for asylum seekers and refugees in the South-Eastern Region of Melbourne: Monash Health Refugee Health and Wellbeing. Australian Journal of Primary Health 23, 323-328. doi:10.1071/PY16092

Milosevic D, Cheng IH, Smith MM (2012) The NSW Refugee Health Service - improving refugee access to primary care. Australian Family Physician 41, 147-149.

Owens C, Dandy J, Hancock P (2016) Perceptions of pregnancy experiences when using a community-based antenatal service: a qualitative study of refugee and migrant women in Perth, Western Australia. Women and Birth 29, 128-137. doi:10.1016/j.wombi.2015.09.003

Russell GM, Long K, Lewis V, Enticott JC, Gunatillaka N, Cheng IH, Marsh G, Vasi S, Advocat J, Saito S, Song H, Casey S, Smith M, Harris MF (2021) OPTIMISE: a pragmatic stepped wedge cluster randomised trial of an intervention to improve primary care for refugees in Australia. The Medical Journal of Australia 215, 420-426. doi:10.5694/mja2.51278

Sackey D, Jones M, Farley R (2020) Reconceptualising specialisation: integrating refugee health in primary care. Australian Journal of Primary Health 26, 452-457. doi:10.1071/PY20138

Timlin M, Russo A, McBride J (2020) Building capacity in primary health care to respond to the needs of asylum seekers and refugees in Melbourne, Australia: the 'GP Engagement' initiative. Australian Journal of Primary Health 26, 10-16. doi:10.1071/PY18190

Valibhoy MC, Kaplan I, Szwarc J (2017) "It comes down to just how human someone can be": a qualitative study with young people from refugee backgrounds about their experiences of Australian mental health services. Transcultural Psychiatry 54, 23-45. doi:10.1177/1363461516662810

Wei K, Chopra P, Strehlow S, Stow M, Kaplan I, Szwarc J, Minas H (2021) The capacity-building role of community liaison workers with refugee communities in Victoria, Australia. International Journal of Mental Health Systems 15, 64. doi:10.1186/s13033-021-00485-9 